\title{
The Representations of the Automorphism Groups and the Frobenius Invariants of K3 Surfaces
}

\author{
JUNMYEONG JANG
}

\begin{abstract}
For a complex algebraic K3 surface, it is known that the representations of the automorphism group on the transcendental cycles is finite and is isomorphic to the representation on the two-forms. In this paper, we prove similar results for a K3 surface defined over a field of odd characteristic. Also, we prove that the height and the Artin invariant of a K3 surface equipped with a nonsymplectic automorphism of some high order are determined by a congruence class of the base characteristic.
\end{abstract}

\section{Introduction}

When $X$ is an algebraic complex $\mathrm{K} 3$ surface, the second integral singular cohomology $H^{2}(X, \mathbb{Z})$ is a free Abelian group of rank 22 equipped with a lattice structure isomorphic to $U^{3} \oplus E_{8}^{2}$. Here $U$ is the hyperbolic plane, and $E_{8}$ is the unique unimodular, even, and negative definite lattice of rank 8 . The cycle map gives a primitive embedding of the Neron-Severi group of $X$ into the second cohomology $N S(X) \hookrightarrow H^{2}(X, \mathbb{Z})$. The rank of $N S(X)$ is called the Picard number of $X$ and is denoted by $\rho(X)$. The orthogonal complement of this embedding is called the transcendental lattice of $X$ and is denoted by $T(X)$. The rank of the transcendental lattice is $22-\rho(X)$. Cohomology $H^{2}(X, \mathbb{Z})$ is an overlattice of $N S(X) \oplus T(X)$, and

$$
\left|H^{2}(X, \mathbb{Z}) /(N S(X) \oplus T(X))\right|=|d(N S(X))| .
$$

The one-dimensional complex space of global holomorphic two-forms of $X$, $H^{0}\left(X, \Omega_{X, \mathbb{C}}^{2}\right)$ is a direct factor of $H^{2}(X, \mathbb{Z}) \otimes \mathbb{C}=H^{2}(X, \mathbb{C})$, and by the Lefschetz $(1,1)$ theorem,

$$
N S(X)=H^{0}\left(X, \Omega_{X, \mathbb{C}}^{2}\right)^{\perp} \cap H^{2}(X, \mathbb{Z})
$$

in $H^{2}(X, \mathbb{C})$. In particular, $H^{0}\left(X, \Omega_{X / \mathbb{C}}^{2}\right)$ is a direct factor of $T(X) \otimes \mathbb{C}$. The automorphism group of $X, \operatorname{Aut}(X)$, has natural actions on $T(X)$ and on $H^{0}\left(X, \Omega_{X / \mathbb{C}}^{2}\right)$. Let us denote the actions of $\operatorname{Aut}(X)$ on the transcendental lattice and the two-forms by

$$
\chi_{X}: \operatorname{Aut}(X) \rightarrow O(T(X)) \quad \text { and } \quad \rho_{X}: \operatorname{Aut}(X) \rightarrow G l\left(H^{0}\left(X, \Omega_{X / \mathbb{C}}^{2}\right)\right) .
$$

Received January 5, 2015. Revision received June 16, 2015.

This research was supported by Basic Science Research Program through the National Research Foundation of Korea (NRF) funded by the Ministry of Education (2015R1D1A1A01058962) and KIAS grant funded by the Korea government. 
We say that an automorphism of $X, \alpha: X \rightarrow X$ is symplectic if $\rho_{X}(\alpha)=1$. If $\alpha$ is of finite order greater than 1 and the order of $\alpha$ is equal to the order of $\rho_{X}(\alpha)$, we say that $\alpha$ is purely nonsymplectic. Since $H^{0}\left(X, \Omega_{X / \mathbb{C}}^{2}\right)$ is a direct factor of $T(X) \otimes \mathbb{C}$, there is a canonical surjection $p_{X}: \operatorname{Im} \chi_{X} \rightarrow \operatorname{Im} \rho_{X}$. It is known that $p_{X}$ is an isomorphism and $\operatorname{Im} \chi_{X}$ and $\operatorname{Im} \rho_{X}$ are finite cyclic groups [21]. The proof of this result is based on the Lefschetz $(1,1)$ theorem and the Torelli theorem for $\mathrm{K} 3$ surfaces. If the order of $\operatorname{Im} \rho_{X}$ is $N$, then there is an automorphism $\alpha \in$ Aut $X$ such that $\xi_{N}=\rho_{X}(\alpha)$ is a primitive $N$ th root of unity. Then $T(X)$ has a free $\mathbb{Z}\left[\xi_{N}\right]$-module structure in a natural way [18], and $22-\rho(X)$ is a multiple of $\phi(N)$. Here $\phi$ is the Euler $\phi$-function.

Assume that $k$ is an algebraically closed field of odd characteristic $p$ and $W$ is the ring of Witt vectors of $k$. Let $X$ be a K3 surface defined over $k$. The second crystalline cohomology $H_{\text {cris }}^{2}(X / W)$ and the second étale cohomology $H_{\text {ét }}^{2}\left(X, \mathbb{Z}_{l}\right)$ are unimodular lattices of rank 22 over $W$ and $\mathbb{Z}_{l}$, respectively. Here $l$ is a prime number different from $p$. The cycle maps to the crystalline cohomology and étale cohomology give an embedding of $W$-modules

$$
N S(X) \otimes W \hookrightarrow H_{\text {cris }}^{2}(X / W)
$$

and an embedding of $\mathbb{Z}_{l}$-modules

$$
N S(X) \otimes \mathbb{Z}_{l} \hookrightarrow H_{\text {ét }}^{2}\left(X, \mathbb{Z}_{l}\right) .
$$

The Newton polygon of $H_{\text {cris }}^{2}(X / W)$ is determined by the height of the formal Brauer group of $X$ (see Section 2). This height is a positive integer between 1 and 10 or $\infty$. If the height of $X$ is $\infty$, then we say that $X$ is supersingular. We again denote the representation of the automorphism group of $X$ on the global two-forms by

$$
\rho_{X}: \operatorname{Aut}(X) \rightarrow G l\left(H^{0}\left(X, \Omega_{X / k}^{2}\right)\right) .
$$

When $X$ is a supersingular K3 surface over $k, \rho(X)$ is 22 [20; 5; 19], and the discriminant group of the Neron-Severi group is $(\mathbb{Z} / p)^{2 \sigma}$ for a positive integer $\sigma$ between 1 and 10 . We say that $\sigma$ is the Artin-invariant of $X$. By the Frobenius invariant of a K3 surface in positive characteristic we mean the height and the Artin-invariant. If the Artin-invariant of $X$ is $\sigma$, then $H_{\text {cris }}^{2}(X / W) /(N S(X) \otimes W)$ is a $\sigma$-dimensional $k$-space, and there is a canonical projection

$$
H_{\text {cris }}^{2}(X / W) /(N S(X) \otimes W) \rightarrow H^{2}\left(X, \mathcal{O}_{X}\right) .
$$

Moreover, $H_{\text {cris }}^{2}(X / W) /(N S(X) \otimes W)$ is an invariant isotropic subspace of the discriminant group $\left(N S(X)^{*} / N S(X)\right) \otimes k$. Let

$$
v_{X}: \operatorname{Aut}(X) \rightarrow O\left(N S(X)^{*} / N S(X)\right)
$$

be the representations on the discriminant group of the Neron-Severi group. We prove that there is a canonical isomorphism $\operatorname{Im} v_{X} \rightarrow \operatorname{Im} \rho_{X}$ and $\operatorname{Im} v_{X} \simeq \operatorname{Im} \rho_{X}$ is a finite cyclic group (Prop. 3.1).

When $X$ is a K3 surface of finite height $h$ over $k, \rho(X)$ is at most $22-2 h$ [3]. For a K3 surface of finite height $X$, we call the orthogonal complements of the 
cycle maps

$$
N S(X) \otimes \mathbb{Z}_{l} \hookrightarrow H_{\text {ét }}^{2}\left(X, \mathbb{Z}_{l}\right) \quad \text { and } \quad N S(X) \otimes W \hookrightarrow H_{\text {cris }}^{2}(X / W)
$$

the $l$-adic transcendental lattice of $X$ and the crystalline transcendental lattice of $X$, respectively. We denote those lattices by $T_{l}(X)$ and $T_{\text {cris }}(X)$. The representation of $\operatorname{Aut}(X)$ on $T_{l}(X)$ and $T_{\text {cris }}(X)$ are denoted by

$$
\chi_{l, X}: \operatorname{Aut}(X) \rightarrow O\left(T_{l}(X)\right) \quad \text { and } \quad \chi_{\text {cris }, X}: \operatorname{Aut}(X) \rightarrow O\left(T_{\text {cris }}(X)\right) .
$$

We will see that $\operatorname{ker} \chi_{l, X}$ is equal to $\operatorname{ker} \chi_{X \text {,cris }}$ and for any automorphism $\alpha \in$ $\operatorname{Aut}(X)$, the characteristic polynomial of $\chi_{l, X}(\alpha)$ is equal to the characteristic polynomial of $\chi_{\text {cris }, X}(\alpha)$ (Prop. 3.6). We will also construct canonical projections

$$
p_{\text {cris }, X}: \operatorname{Im} \chi_{\text {cris }, X} \rightarrow \operatorname{Im} \rho_{X} \quad \text { and } \quad p_{l, X}: \operatorname{Im} \chi_{l, X} \rightarrow \operatorname{Im} \rho_{X}
$$

satisfying $\rho_{X}=p_{\text {cris }, X} \circ \chi_{\text {cris }, X}=p_{l, X} \circ \chi_{l, X}$. Also, using a Neron-Severi group preserving lifting of $X$, we prove that $\operatorname{Im} \chi_{\text {cris }, X}, \operatorname{Im} \chi_{l, X}$, and $\operatorname{Im} \rho_{X}$ are finite (Props. 3.5 and 3.6). It follows that, for any $\alpha \in \operatorname{Aut}(X)$, all the eigenvalues of $\chi_{l, X}(\alpha)$ are roots of unity. In addition to that, if the order of $\operatorname{Im} \chi_{l, X}(\alpha)$ is not divisible by $p$ and the order of $\rho_{X}(\alpha)$ is $n$, every primitive $n$th root of unity occurs as an eigenvalue of $\chi_{l, X}(\alpha)$ (Prop. 3.7). This generalizes Proposition 2.1 in [13].

When $\alpha$ is an automorphism of a K3 surface $X$ over $k$, under certain conditions, some parts of eigenvalues of $\alpha^{*} \mid H_{\text {ét }}^{2}\left(X, \mathbb{Q}_{l}\right)$ are decided by the Frobenius invariant of $X$ and $\rho_{X}(\alpha)$. More precisely, we have the following result.

THEOREM 3.9. Let $k$ be an algebraically closed field of odd characteristic $p$. Assume that $X$ is a K3 surface over $k$ and $\alpha$ is an automorphism of $X$. We assume either of the following:

(1) $X$ is of finite height $h$, and the order of $\chi_{l, X}(\alpha)$ is prime to $p$

or

(2) $X$ is supersingular of Artin-invariant $\sigma$, and the order of $\alpha$ is finite and prime to $p$.

Suppose that $\rho_{X}(\alpha)(u)=\zeta \cdot u$ for a generator $u$ of $H^{0}\left(X, \Omega_{X / k}^{2}\right)$ and that $\xi$ is the Teichmüller lift of $\zeta$ in $W$.

Then in case (1), $\xi^{ \pm p^{0}}, \xi^{ \pm p^{-1}}, \ldots, \xi^{ \pm p^{1-h}}$ appear as eigenvalues of $\chi_{l, X}(\alpha)$ and in case (2), $\xi^{ \pm p^{0}}, \xi^{ \pm p^{-1}}, \ldots, \xi^{ \pm p^{1-\sigma}}$ appear as eigenvalues of $\alpha^{*} \mid H_{\text {ett }}^{2}\left(X, \mathbb{Q}_{l}\right)$.

Based on Theorem 3.9 and the Tate conjecture for K3 surfaces of finite height $[23 ; 19]$, we can prove the followings.

THEOREM 3.10. Let $k$ be an algebraic closure of a finite field of odd characteristic $p$, and $X$ be a K3 surface of finite height $h$ over $k$. If the order of $\operatorname{Im} \chi_{l, X}$ is not divisible by $p$, the projection $p_{l, X}: \operatorname{Im} \chi_{X, l} \rightarrow \operatorname{Im} \rho_{X}$ is bijective.

COROLlARY 3.11. Let $k$ be an algebraic closure of a finite field of odd characteristic $p$, and $X$ be a K3 surface of finite height over $k$. If $N$ is the order of $\operatorname{Im} \rho_{X}$, then the rank of $T_{l}(X)=22-\rho(X)$ is divisible by $\phi(N)$. 
We can apply these results to study the relation of Frobenius invariant and nonsymplectic automorphisms for K3 surfaces. We prove the followings.

COROLLARY 4.3. Let $k$ be an algebraically closed field of odd characteristic $p$, and $X$ be a K3 surface over $k$. Let $\alpha$ be an automorphism of $X$. We assume that the order of $\rho_{X}(\alpha)$ is $N(>2)$ and that the rank of the Neron-Severi group of $X$ is at least $22-\phi(N)$. If $p^{m} \equiv-1$ modulo $N$ for some $m$, then $X$ is supersingular. If $p^{m} \not \equiv-1$ modulo $N$ for any $m$ and the order of $p$ in $(\mathbb{Z} / N \mathbb{Z})^{*}$ is $n$, then the height of $X$ is $n$.

COROLlary 4.4. Assume that $X$ is a K3 surface over $k$ and $\alpha$ is an automorphism of $X$ such that the order of $\rho_{X}(\alpha)$ is $N(>2)$. We assume that $\alpha$ is of finite order prime to $p$ and that a primitive $N$ th root of unity appears only one time in the eigenvalues of $\alpha^{*} \mid H_{\mathrm{et}}^{2}\left(X, \mathbb{Q}_{l}\right)$. If the order of $p$ in $(\mathbb{Z} / N \mathbb{Z})^{*}$ is $2 n$ and $p^{n} \equiv-1$ modulo $N$, then $X$ is supersingular of Artin-invariant $n$.

If $X$ is a complex algebraic $\mathrm{K} 3$ surface with $N=\left|\operatorname{Im} \rho_{X}\right|$ and the rank of $T(X)$ is equal to $\phi(N)$, then $X$ has a model defined over a number field [26]. By the last two corollaries we deduce that for almost all places, the Frobenius invariant of the reduction of the model of $X$ over the number field is determined by the congruence class of the residue characteristic modulo $N$ (Theorem 4.7). This generalizes the results on the Delsarte K3 surfaces in $[28 ; 30 ; 7]$.

\section{Crystalline Cohomology of K3 Surfaces}

In this section, we review some facts on the Neron-Severi group and the crystalline cohomology of K3 surfaces over a field of odd characteristic. Assume that $k$ is an algebraically closed field of characteristic $p>2$. Let $W$ be the ring of Witt vectors of $k$, and $K$ be the fraction field of $W$. Assume that $X$ is a K3 surface over $k$. Let $\widehat{B r}_{X}$ be the formal Brauer group of $X . \widehat{B r}_{X}$ is a smooth formal group of dimension 1 over $k$ [2]. A smooth formal group of dimension 1 over an algebraically closed filed of positive characteristic is classified by its height. The height $h$ of $\widehat{B r}_{X}$ is a positive integer $(1 \leq h \leq 10)$ or $\infty$. When $h=\infty$, we say $X$ is supersingular. The Dieudonné module of $\widehat{\widehat{B r}}_{X}$ is

$$
\mathbb{D}\left(\widehat{B r}_{X}\right)=W[F, V] /\left(F V=p, F=V^{h-1}\right)
$$

if $h$ is finite or

$$
\mathbb{D}\left(\widehat{B r}_{X}\right)=k[[V]]
$$

if $h=\infty$. Here $F$ is a Frobenius linear operator, and $V$ is a Frobenius inverse linear operator.

The crystalline cohomologies $H_{\text {cris }}^{i}(X / W)$ are finite free $W$-modules of ranks 1, 0, 22, 0,1 for $i=0,1,2,3,4$, respectively, equipped with Frobenius linear operators

$$
\mathbf{F}: H_{\text {cris }}^{i}(X / W) \rightarrow H_{\text {cris }}^{i}(X / W) .
$$


If the height $h$ is finite, then the Frobenius slopes of $H_{\text {cris }}^{2}(X / W)$ are $1-$ $1 / h, 1,1+1 / h$ of lengths $h, 22-2 h, h$, respectively. If $X$ is supersingular, then the only Frobenius slope of $H_{\text {cris }}^{2}(X / W)$ is 1 of length 22.

The crystalline cohomology $H_{\text {cris }}^{i}(X / W)$ can be realized as the hypercohomology of the DeRham-Witt complex [9],

$$
0 \rightarrow W \mathcal{O}_{X} \rightarrow W \Omega_{X / k}^{1} \rightarrow W \Omega_{X / k}^{2} \rightarrow 0 .
$$

The naive filtration of the DeRham-Witt complex gives the slope spectral sequence

$$
H^{i}\left(X, W \Omega_{X / k}^{j}\right) \Rightarrow H_{\text {cris }}^{i+j}(X / W) .
$$

The $E_{1}$-level page of the slope spectral sequence of $X$ is

$$
\begin{aligned}
& H^{2}\left(X, W \mathcal{O}_{X}\right) \stackrel{d}{\rightarrow} H^{2}\left(X, W \Omega_{X}^{1}\right) \rightarrow \quad W \\
& 0 \quad \rightarrow H^{1}\left(X, W \Omega_{X}^{1}\right) \rightarrow \quad 0 \\
& W \quad \rightarrow \quad 0 \quad \rightarrow H^{0}\left(X, W \Omega_{X}^{2}\right) \text {. }
\end{aligned}
$$

Here $H^{2}\left(X, W \mathcal{O}_{X}\right)$ is isomorphic to $\mathbb{D}\left(\widehat{B r}_{X}\right)$ [3]. By an exact sequence of sheaves on $X$,

$$
0 \rightarrow W \mathcal{O}_{X} \stackrel{V}{\rightarrow} W \mathcal{O}_{X} \rightarrow \mathcal{O}_{X} \rightarrow 0
$$

we have an isomorphism $H^{2}\left(X, W \mathcal{O}_{X}\right) / V \simeq H^{2}\left(X, \mathcal{O}_{X}\right)$.

If $X$ is of finite height $h$, then $H^{2}\left(X, W \Omega_{X}^{1}\right)=0$, and the slope spectral sequence degenerates at $E_{1}$-level. Moreover, $H_{\text {cris }}^{2}(X / W)$ has an F-crystal decomposition [9], II.7.2, [12], Theorem 1.6.1,

$$
H^{2}(X / W)=H_{\text {cris }}^{2}(X / W)_{[1-1 / h]} \oplus H_{\text {cris }}^{2}(X / W)_{[1]} \oplus H_{\text {cris }}^{2}(X / W)_{[1+1 / h]} .
$$

Here

and

$$
H_{\text {cris }}^{2}(X / W)_{[1-1 / h]}=H^{2}\left(X, W \mathcal{O}_{X}\right)=\mathbb{D}\left(\widehat{B r}_{X}\right)
$$

$$
H_{\text {cris }}^{2}(X / W)_{[1+1 / h]}=\operatorname{Hom}\left(H^{2}\left(X, W \mathcal{O}_{X}\right), H^{4}(X / W)\right) .
$$

Note that $H^{4}(X / W)$ is a free $W$-module of rank 1 equipped with a Frobenius linear operator of slope 2 . For the cup product pairing on $H_{\text {cris }}^{2}(X / W)$, $H_{\text {cris }}^{2}(X / W)_{[1-1 / h]}$ and $H_{\text {cris }}^{2}(X / W)_{[1+1 / h]}$ are isotropic and dual to each other. On the other hand, $H_{\text {cris }}^{2}(X / W)_{[1]}$ is unimodular. The discriminant of the $\mathbb{Z}_{p^{-}}$ lattice $H_{\text {cris }}^{2}(X / W)_{[1]}^{F=p}$ is $(-1)^{h+1}$. When $X$ is of finite height $h$, the Frobenius morphism and the lattice structure of $H_{\text {cris }}^{2}(X / W)$ are completely determined by $h$ ([25], p. 363). Because there exists a canonical embedding ([9], Proposition II.5.12),

$$
N S(X) \otimes \mathbb{Z}_{p} \hookrightarrow H^{1}\left(X, W \Omega_{X}^{1}\right)^{F=p},
$$

the Picard number $\rho(X)$ of $X$ is not greater than the length of slope 1 part of $H_{\text {cris }}^{2}(X / W)$. It follows that $\rho(X) \leq 22-2 h$ if $h$ is finite.

In odd characteristic, it is known that $X$ is supersingular if and only if the Picard number of $X$ is $22[20 ; 5 ; 19]$. Assume that $X$ is a supersingular K3 surface. The discriminant of $N S(X)$ is $-p^{2 \sigma}$ for an integer $\sigma$ between 1 and 10 , and $\sigma$ is called the Artin-invariant of $X$. The discriminant group of $N S(X)$ is isomorphic 
to $(\mathbb{Z} / p)^{2 \sigma}$. Moreover, $N S(X)$ is determined by the base characteristic $p$ and $\sigma$ [27].

For a supersingular $\mathrm{K} 3$ surface $X, H^{0}\left(X, W \Omega_{X}^{2}\right)=0$, and the slope spectral sequence degenerates at $E_{2}$-level ([9], Corollaire II.3.13). The only nontrivial map in the $E_{1}$-page of the slope spectral sequence is

$$
d: H^{2}\left(X, W \mathcal{O}_{X}\right) \rightarrow H^{2}\left(X, W \Omega_{X}^{1}\right) .
$$

Here $d$ is surjective, and

$$
\operatorname{ker} d=H_{\text {cris }}^{2}(X / W) / F^{1} H_{\text {cris }}^{2}(X / W),
$$

where $F^{\cdot} H_{\text {cris }}^{2}(X / W)$ is the filtration given by the slope spectral sequence. We can identify $F^{1} H_{\text {cris }}^{2}(X / W)$ with the image of the cycle map ([9], II.7.2)

$$
N S(X) \otimes W \hookrightarrow H_{\text {cris }}^{2}(X / W) .
$$

Since $H_{\text {cris }}^{2}(X / W)$ is a unimodular $W$-lattice and the cycle map preserves the paring, we have a chain

$$
N S(X) \otimes W \subset H_{\text {cris }}^{2}(X / W) \subset\left(N S(X)^{*}\right) \otimes W .
$$

Moreover, $\operatorname{ker} d=H_{\text {cris }}^{2}(X / W) /(N S(X) \otimes W)$ is a $\sigma$-dimensional isotropic $k$ subspace of the discriminant group $\left(N S(X)^{*} \otimes W\right) /(N S(X) \otimes W)=$ $\left(N S(X)^{*} / N S(X)\right) \otimes k$. It is also known that

$$
\operatorname{ker} d V^{i}: H^{2}\left(X, W \mathcal{O}_{X}\right) \rightarrow H^{2}\left(X, W \Omega_{X}^{1}\right)
$$

is a $(\sigma-i)$-dimensional $k$-space for $i \leq \sigma$ and $\operatorname{ker} d V^{i+1} \subseteq \operatorname{ker} d V^{i}$ ([22], Thm. $0.1)$. When $x$ is a nonzero element of $\operatorname{ker} d V^{\sigma-1}$,

$$
x, V x, \ldots, V^{\sigma-i-1} x
$$

generate $\operatorname{ker} V^{i} d$ over $k$, and $x$ is a $V$-adic topological generator of $H^{2}\left(X, W \mathcal{O}_{X}\right)$. The composition

$$
\operatorname{ker} d V^{\sigma-1} \hookrightarrow H^{2}\left(X, W \mathcal{O}_{X}\right) \rightarrow H^{2}\left(X, \mathcal{O}_{X}\right)
$$

is an isomorphism.

\section{Representations of the Automorphism Groups on the Two-Forms and Transcendental Cycles}

Let $k$ be an algebraically closed field of characteristic $p>2$. Let $W$ be the ring of Witt vectors of $k$, and $K$ be the fraction field of $W$. Let $X$ be a K3 surface over $k$. Let

$$
\rho_{X}: \operatorname{Aut}(X) \rightarrow G L\left(H^{0}\left(X, \Omega_{X / k}^{2}\right)\right) \quad \text { and } \quad \lambda_{X}: \operatorname{Aut}(X) \rightarrow G L\left(H^{2}\left(X, \mathcal{O}_{X}\right)\right)
$$

be the representation of $\operatorname{Aut}(X)$ on $H^{0}\left(X, \Omega_{X / k}^{2}\right)$ and $H^{2}\left(X, \mathcal{O}_{X}\right)$. By the Serre duality, for any $\alpha \in$ Aut $X, \rho_{X}(\alpha)^{-1}=\lambda_{X}(\alpha)$ and $\operatorname{ker} \rho_{X}=\operatorname{ker} \lambda_{X}$.

Assume that $X$ is supersingular. Let

$$
v_{X}: \operatorname{Aut}(X) \rightarrow O\left(\left(N S(X)^{*} / N S(X)\right) \otimes k\right)
$$


be the representation of $\operatorname{Aut}(X)$ on the discriminant group $\left(N S(X)^{*} / N S(X)\right) \otimes k$. Because $v_{X}$ factors through the action of $\operatorname{Aut}(X)$ on $\left(N S(X)^{*} / N S(X)\right)$, a finitedimensional space over a finite field $\mathbb{Z} / p, \operatorname{Im} v_{X}$ is finite. Since ker $d: H^{2}(X$, $\left.W \mathcal{O}_{X}\right) \rightarrow H^{2}\left(X, W \Omega_{X}^{1}\right)$ is an invariant subspace of $\left(N S(X)^{*} / N S(X)\right) \otimes k$ for the action of Aut $X$ and there is a projection $\operatorname{ker} d \rightarrow H^{2}\left(X, \mathcal{O}_{X}\right)$, we have a surjective map $q_{X}: \operatorname{Im} v_{X} \rightarrow \operatorname{Im} \lambda_{X}$ such that $q_{X} \circ v_{X}=\lambda_{X}$. Then a surjective $\operatorname{map} p_{X}=q_{X}^{-1}: \operatorname{Im} v_{X} \rightarrow \operatorname{Im} \rho_{X}$ satisfies $p_{X} \circ v_{X}=\rho_{X}$.

Proposition 3.1. Let $X$ be a supersingular K3 surface in odd characteristic. Then $p_{X}: \operatorname{Im} v_{X} \rightarrow \operatorname{Im} \rho_{X}$ is an isomorphism.

Proof. Let $\sigma$ be the Artin-invariant of $X$, and $x$ be a nonzero element of $\operatorname{ker} d V^{\sigma-1}$. Then, $x_{i}=V^{i} x, i=1, \ldots, \sigma-1$, is a basis of ker $d$. Let $y_{i}$ be the dual basis for $x_{i}$ of the dual isotropic subspace of ker $d$ in $\left(N S(X)^{*} / N S(X)\right) \otimes k$. Then any automorphism $\alpha \in \operatorname{Aut}(X)$ preserves all the lines $k \cdot x_{i}$ and $k \cdot y_{i}$. In other words, all $x_{i}$ and $y_{i}$ are eigenvectors of $v_{X}(\alpha)$. Since $\alpha^{*}\left(V^{i} x\right)=V^{i} \alpha^{*}(x)$ and $y_{i}$ is dual to $x_{i}, v_{X}(\alpha)$ is decided by the eigenvalue at $x=x_{0}$. But, for any $\alpha \in \operatorname{Aut}(X), \rho(\alpha)$ is the inverse of the eigenvalue of $v_{X}(\alpha)$ for an eigenvector $x_{0}$, so $p_{X}$ is injective.

REMARK 3.2. In [16], a supersingular K3 surface is defined to be generic if the order of $\operatorname{Im} v_{X}$ is 1 or 2 . Also, it is proved that there exists a generic supersingular $\mathrm{K} 3$ surface of Artin-invariant $\sigma \geq 2$ in odd characteristic ([16], Thm. 1.7). By the last proposition, a supersingular $\mathrm{K} 3$ surface in odd characteristic is generic if and only if the order of $\operatorname{Im} \rho_{X}$ is 1 or 2 .

For the order of the $\operatorname{Im} \rho$, the following is known.

Proposition 3.3 ([22], Thm. 2.1). The cardinality of $\operatorname{Im} \rho_{X}$ divides $p^{\sigma}+1$.

REMARK 3.4. If $X$ is a supersingular K3 surface of Artin-invariant 1, then we have shown, using the crystalline Torelli theorem, that $\operatorname{Im} \rho_{X} \simeq \operatorname{Im} v_{X}$ is a cyclic group of rank $p+1$ ([11], Thm. 3.3). By this result, if $\phi(p+1)>20$, then $X$ has an automorphism that cannot be lifted to characteristic 0 .

Now we assume that $X$ is a K3 surface of finite height over $k$. There is a smooth lifting of $X$ over $W, \mathcal{X} / W$, with the generic fiber $\mathcal{X}_{K}=\mathcal{X} \otimes K$ such that the reduction map

$$
N S\left(\mathcal{X}_{K}\right) \rightarrow N S(X)
$$

is an isomorphism $[23 ; 17 ; 10]$. We say that a lifting of $X$ satisfying this condition is a Neron-Severi group preserving lifting of $X$. When $\mathcal{X}$ is a Neron-Severi group preserving lifting and $\bar{K}$ is an algebraic closure of $K$, the specialization map

$$
\operatorname{Aut}\left(\mathcal{X}_{K} \otimes \bar{K}\right) \rightarrow \operatorname{Aut}(X)
$$

is an injection of finite index ([17], Thm. 6.1). 
Proposition 3.5. Let $X$ be a K3 surface of finite height over $k$. Then $\operatorname{Im} \rho_{X}$ is finite.

Proof. Let $\mathcal{X} / W$ be a Neron-Severi group preserving lifting of $X$, and $\mathcal{X}_{K} / K$ be the generic fiber of $\mathcal{X} / W$. Since $K$ is of characteristic 0 , the image of $\operatorname{Aut}\left(\mathcal{X}_{\bar{K}}\right) \rightarrow$ $G L\left(H^{0}\left(\mathcal{X}_{\bar{K}}, \Omega_{\mathcal{X}_{\bar{K}} / \bar{K}}^{2}\right)\right)$ is finite. Therefore, the image of $\operatorname{Aut}\left(\mathcal{X}_{\bar{K}}\right) \hookrightarrow \operatorname{Aut}(X) \rightarrow$ $G L\left(H^{0}\left(X, \Omega_{X / k}^{2}\right)\right)$ is also finite. Since $\operatorname{Aut}\left(\mathcal{X}_{\bar{K}}\right)$ is of finite index in $\operatorname{Aut}(X)$, the image of $\rho_{X}$ is finite.

Let $X$ be a K3 surface of finite height over $k$. Let $T_{l}(X)$ be the orthogonal complement of the cycle map

$$
N S(X) \otimes \mathbb{Z}_{l} \hookrightarrow H_{\text {ett }}^{2}\left(X, \mathbb{Z}_{l}\right)
$$

for $l \neq p$, and $T_{\text {cris }}(X)$ be the orthogonal complement of the cycle map

$$
N S(X) \otimes W \hookrightarrow H_{\text {cris }}^{2}(X / W) .
$$

We say $T_{l}(X)$ and $T_{\text {cris }}(X)$ are the $l$-adic transcendental lattice of $X$ and the crystalline transcendental lattice of $X$, respectively. When $\rho(X)$ is the Picard number of $X$, the ranks of $T_{l}(X)$ and $T_{\text {cris }}(X)$ are $22-\rho(X)$. Note that

$$
H^{2}\left(X, W \mathcal{O}_{X}\right) \oplus H_{\text {cris }}^{2}(X / W)_{[1+1 / h]}
$$

is a direct factor of $T_{\text {cris }}(X)$ and $H^{2}\left(X, W \mathcal{O}_{X}\right) / V \simeq H^{2}\left(X, \mathcal{O}_{X}\right)$. Hence, there is a canonical projection $T_{\text {cris }}(X) \rightarrow H^{2}\left(X, \mathcal{O}_{X}\right)$. Let

$$
\chi_{l, X}: \operatorname{Aut}(X) \rightarrow O\left(T_{l}(X)\right) \quad \text { and } \quad \chi_{\text {cris }, X}: \operatorname{Aut}(X) \rightarrow O\left(T_{\text {cris }}(X)\right)
$$

be the canonical representations.

Proposition 3.6. Let $X$ be a K3 surface of finite height over $k$. The images of $\chi_{l, X}$ and $\chi_{\text {cris, } X}$ are finite, and there is an isomorphism $\psi_{l}: \operatorname{Im} \chi_{l, X} \rightarrow \operatorname{Im} \chi_{\text {cris }, X}$ such that $\psi_{l} \circ \chi_{l, X}=\chi_{\text {cris }, X}$.

Proof. Let $\mathcal{X} / W$ be a Neron-Severi group preserving lifting of $X$ with the generic fiber $\mathcal{X}_{K}=\mathcal{X} \otimes K$. $\operatorname{Aut}\left(\mathcal{X}_{\bar{K}}\right)$ is a subgroup of $\operatorname{Aut}(X)$ of finite index. If we identify $H_{\text {ét }}^{2}\left(\mathcal{X}_{\bar{K}}, \mathbb{Z}_{l}\right)$ with $H_{\text {ét }}^{2}\left(X, \mathbb{Z}_{l}\right), T_{l}(X)$ is equal to the orthogonal complement of the cycle map

$$
N S\left(\mathcal{X}_{\bar{K}}\right) \otimes \mathbb{Z}_{l} \hookrightarrow H_{\text {ét }}^{2}\left(\mathcal{X}_{\bar{K}}, \mathbb{Z}_{l}\right)
$$

Because $\bar{K}$ is of characteristic 0 , the action of $\operatorname{Aut}\left(\mathcal{X}_{\bar{K}}\right)$ on $T_{l}(X)$ has a finite image. Therefore, $\operatorname{Im} \chi_{l, X}$ is finite. In a similar way, there is a canonical isomorphism

$$
H_{d r}^{2}\left(\mathcal{X}_{\bar{K}} / \bar{K}\right) \simeq H_{\text {cris }}^{2}(X / W) \otimes \bar{K}
$$

which is compatible with the action of $\operatorname{Aut}\left(\mathcal{X}_{\bar{K}}\right)$ on both sides ([4, Cor. 2.5]). Also, this isomorphism is compatible with two cycle maps (loc. cit., Cor. 3.7),

$$
N S\left(\mathcal{X}_{\bar{K}}\right) \rightarrow H_{d r}^{2}\left(\mathcal{X}_{\bar{K}} / \bar{K}\right) \quad \text { and } \quad N S(X) \rightarrow H_{\text {cris }}^{2}(X / W) \otimes \bar{K}
$$


It follows that the action of $\operatorname{Aut}\left(\mathcal{X}_{\bar{K}}\right)$ on $T_{\text {cris }}(X)$ has a finite image, and so does the action of $\operatorname{Aut}(X)$ on $T_{\text {cris }}(X)$. When $\alpha$ is an automorphism of $X$, the characteristic polynomials of $\alpha^{*} \mid H_{\text {cris }}^{2}(X / K)$ and $\alpha^{*} \mid H_{\text {et }}^{2}\left(X, \mathbb{Q}_{l}\right)$ are equal to each other and have integer coefficients ([8], 3.7.3). Note that the characteristic polynomial of $\alpha^{*} \mid H_{e t}^{2}\left(X, \mathbb{Q}_{l}\right)$ is the product of the characteristic polynomial of $\alpha^{*} \mid N S(X)$ and the characteristic polynomial of $\chi_{l, X}(\alpha)$. Also, the characteristic polynomial of $\alpha^{*} \mid H_{\text {cris }}^{2}(X / K)$ is the product of the characteristic polynomial of $\alpha^{*} \mid N S(X)$ and the characteristic polynomial of $\chi_{\text {cris }, X}(\alpha)$. Because $N S(X)$ is an integral lattice, the characteristic polynomial of $\alpha^{*} \mid N S(X)$ is also integral, and the characteristic polynomials of $\chi_{l, X}(\alpha)$ and $\chi_{\text {cris, } X}(\alpha)$ are equal to each other and integral. Since $\chi_{l, X}(\alpha)$ and $\chi_{\text {cris }, X}(\alpha)$ are of finite orders, they are semisimple, and all their eigenvalues are roots of unity. It follows that $\chi_{l, X}(\alpha)=i d$ if and only if $\chi_{\text {cris }, X}(\alpha)=i d$. Therefore, $\operatorname{ker} \chi_{l, X}=\operatorname{ker} \chi_{\text {cris, } X}$, and there exists a compatible isomorphism $\psi_{l}: \operatorname{Im} \chi_{l, X} \rightarrow \operatorname{Im} \chi_{\text {cris }, X}$.

Using the projection $T_{\text {cris }}(X) \rightarrow H^{2}\left(X, \mathcal{O}_{X}\right)$ and the Serre duality, we have a canonical projection $p_{\text {cirs }, X}: \operatorname{Im} \chi_{\text {cris }, X} \rightarrow \operatorname{Im} \rho_{X}$ such that $p_{\text {cris }, X} \circ \chi_{\text {cris }, X}=$ $\rho_{X}$. Composing with $\psi_{l}$, we have a canonical projection $p_{l, X}=p_{\text {cris }, X} \circ \psi_{l}$ : $\operatorname{Im} \chi_{l, X} \rightarrow \operatorname{Im} \rho_{X}$.

Proposition 3.7. Let $X$ be a K3 surface of finite height, and $\alpha$ be an automorphism of $X$. If the order of $\chi_{l, X}(\alpha)$ is prime to $p$ and the order of $\rho_{X}(\alpha)$ is $n$, then all the primitive $n$th roots of unity appear as eigenvalues of $\chi_{\mathrm{cris}, X}(\alpha)$.

Proof. Let $\zeta=\rho_{X}(\alpha) \in k^{*}$, and let $\xi$ be the Teichmüller lifting of $\zeta$ in $W$. Since there is a projection

$$
T_{\text {cris }}(X) / p \rightarrow H^{2}\left(X, \mathcal{O}_{X}\right),
$$

$\zeta^{-1}$ is an eigenvalue of $\alpha^{*} \mid\left(T_{\text {cris }}(X) / p\right)$, and $\xi^{-1}$ is an eigenvalue of $\chi_{\text {cris, } X}(\alpha)$. Because the characteristic polynomial of $\chi_{\text {cris, } X}(\alpha)$ is integral and $\xi^{-1}$ is a primitive $n$th root of unity, the $n$th cyclotomic polynomial divides the characteristic polynomial of $\chi_{\text {cris, } X}(\alpha)$. Therefore, every primitive $n$th root of unity is an eigenvalue of $\chi_{\text {cris }, X}(\alpha)$.

REMARK 3.8. Because the rank of the transcendental lattice is not greater than 21 and the degree of the $n$th cyclotomic polynomial is $\phi(n)$, if an $n$th root of unity appears as an eigenvalue of $\chi_{\text {cris, } X}(\alpha)$, then $\phi(n) \leq 21$. Here $\phi$ is the Euler $\phi$ function. In particular, if $p \geq 23$, then a $p$ th root of unity can not appear as an eigenvalue of $\chi_{\text {cris, } X}(\alpha)$.

THEOREM 3.9. Let $k$ be an algebraically closed field of odd characteristic p. Let $X$ be a K3 surface over $k$, and $\alpha$ be an automorphism of $X$. We assume either of the following:

(1) $X$ is of finite height $h$, and the order of $\chi_{l, X}(\alpha)$ is prime to $p$

or

(2) $X$ is supersingular of Artin-invariant $\sigma$, and the order of $\alpha$ is finite and prime to $p$. 
Suppose $\zeta=\rho_{X}(\alpha)$ and $\xi$ is the Teichmüller lift of $\zeta$ in $W$.

Then in case (1), $\xi^{ \pm p^{0}}, \xi^{ \pm p^{-1}}, \ldots, \xi^{ \pm p^{1-h}}$ appear as eigenvalues of $\chi_{\text {cris, } X}(\alpha)$, and in case (2), $\xi^{ \pm p^{0}}, \xi^{ \pm p^{-1}}, \ldots, \xi^{ \pm p^{1-\sigma}}$ appear as eigenvalues of $\alpha^{*} \mid H_{\text {cris }}^{2}(X / W)$.

Proof. First case: $X$ is of finite height. Assume that $X$ is of finite height $h$ and the order of $\chi_{l}(\alpha)$ is prime to $p$. Let us identify $H^{2}\left(X, W \mathcal{O}_{X}\right)$ with $W[F, V] /$ $\left(F V-p, F-V^{h-1}\right)$. Let $f: W \rightarrow W$ be the Frobenius morphism. We assume that

$$
\alpha^{*}(1)=a_{0} 1+a_{1} V+\cdots+a_{h-1} V^{h-1} .
$$

Here $1 \in W[F, V] /\left(F V-p, F-V^{h-1}\right)$ is a $V$-adic topological generator, and $a_{i} \in W$. Note that $a_{0}$ is a unit of $W$. Then

$$
\alpha^{*}\left(V^{i}\right)=V^{i} \alpha^{*}(1)=f^{-i}\left(a_{0}\right) V^{i}+f^{-i}\left(a_{1}\right) V^{i+1}+\cdots+f^{-i}\left(a_{h-1}\right) V^{h+i-1}
$$

for $i \leq h-1$. But $H^{2}\left(X, W \mathcal{O}_{X}\right) / V=H^{2}\left(X, \mathcal{O}_{X}\right)$, so $a_{0} \equiv \zeta^{-1}$ modulo $p$. The matrix of $\alpha^{*} \mid\left(H^{2}\left(X, W \mathcal{O}_{X}\right) / p\right)$ with respect to a basis $1+(p), V+$ $(p), \ldots, V^{h-1}+(p)$ is

$$
\left(\begin{array}{cccc}
\zeta^{-1} & \cdots & \cdots & \cdots \\
0 & \zeta^{-p^{-1}} & \cdots & \cdots \\
\vdots & \vdots & \ddots & \vdots \\
0 & 0 & \cdots & \zeta^{-p^{1-h}}
\end{array}\right)
$$

and the characteristic polynomial of $\alpha^{*} \mid\left(H^{2}\left(X, W \mathcal{O}_{X}\right) / p\right)$ is

$$
\prod_{i=0}^{h-1}\left(T-\zeta^{-p^{-i}}\right)
$$

Since $\alpha^{*} \mid H^{2}\left(X, W \mathcal{O}_{X}\right)$ is of finite order prime to $p$, the characteristic polynomial of $\alpha^{*} \mid H^{2}\left(X, W \mathcal{O}_{X}\right)$ is

$$
\prod_{i=0}^{h-1}\left(T-\xi^{-p^{-i}}\right) .
$$

Because $H_{\text {cris }}^{2}(X / W)_{[1+1 / h]}$ is dual to $H^{2}\left(X, W \mathcal{O}_{X}\right)$, the characteristic polynomial of $\alpha^{*} \mid H_{\text {cris }}^{2}(X / W)_{[1+1 / h]}$ is

$$
\prod_{i=0}^{h-1}\left(T-\xi^{p^{-i}}\right) .
$$

Hence, the claim follows.

Second case: $X$ is supersingular. Assume that $X$ is supersingular of Artininvariant $\sigma$ and $\alpha$ is of finite order prime to $p$. Fix $x_{0}$, a nonzero element of

$$
\operatorname{ker} d V^{\sigma-1}: H^{2}\left(X, W \mathcal{O}_{X}\right) \rightarrow H^{2}\left(X, W \Omega_{X}^{1}\right) \text {. }
$$


Let $x_{i}=V^{i} x$ for $i=0,1, \ldots, \sigma-1$, and let $y_{i}$ be the dual basis of $x_{i}$ in $\left(N S(X)^{*} / N S(X)\right) \otimes k$. Then $\alpha^{*} x_{i}=\zeta^{-p^{-i}} x_{i}$ and $\alpha^{*} y_{i}=\zeta^{p^{-i}} y_{i}$. Because there is an embedding

$$
\left(N S(X)^{*} / N S(X)\right) \otimes k \simeq\left(p N S(X)^{*} / p N S(X)\right) \otimes k \subseteq(N S(X) \otimes W) / p,
$$

$\xi^{ \pm p^{0}}, \xi^{ \pm p^{-1}}, \ldots, \xi^{ \pm p^{1-\sigma}}$ occur as eigenvalues of $\alpha^{*} \mid N S(X) \otimes W$ and so as eigenvalues of $\alpha^{*} \mid N S(X)$. Since $H_{\text {cris }}^{2}(X, W) \otimes K=N S(X) \otimes K$, the claim follows.

When $X$ is a complex algebraic K3 surface, the projection $p_{X}: \operatorname{Im} \chi_{X} \rightarrow \operatorname{Im} \rho_{X}$ is an isomorphism, and the action of $\operatorname{Aut}(X)$ on the transcendental lattice $T(X)$ is determined by the action on $H^{0}\left(X, \Omega_{X / \mathbb{C}}^{2}\right)$, [21]. Moreover, if $N$ is the order of $\operatorname{Im} \rho_{X}$ and $\xi_{N}$ is a primitive $N$ th root of unity, then by the Lefschtz $(1,1)$ theorem $T(X)$ is a torsion-free $\mathbb{Z}\left[\xi_{N}\right]$-module. Because $\phi(N)<22, \mathbb{Z}\left[\xi_{N}\right]$ is a P.I.D. [18], so $T(X)$ is a free $\mathbb{Z}\left[\xi_{N}\right]$-module. It follows that the rank of $T(X)$ is a multiple of $\phi(N)$. We can ask if the same result holds for a K3 surface of finite height in odd characteristic.

THEOREM 3.10. Let $k$ be an algebraic closure of a finite field of odd characteristic $p$, and $X$ be a K3 surface of finite height $h$ over $k$. If the order of $\operatorname{Im} \chi_{l, X}$ is not divisible by $p$, the projection $p_{l, X}: \operatorname{Im} \chi_{l, X} \rightarrow \operatorname{Im} \rho_{X}$ is bijective.

Proof. Clearly, $p_{l, X}$ is surjective. Suppose $X$ is defined over $\mathbb{F}_{q}$ for $q=p^{m}$. The $m$-iterative relative Frobenius morphism of $X / k$ is an endomorphism of $X$ over $k$. We denote this morphism by $F: X \rightarrow X$. The induced morphism $F^{*} \mid H_{\text {ét }}^{2}\left(X, \mathbb{Q}_{l}\right)$ is equal to the Galois action of the geometric Frobenius element in $\operatorname{Gal}\left(k / \mathbb{F}_{q}\right)$ on $H_{\text {ét }}^{2}\left(X, \mathbb{Q}_{l}\right)$. Let $V_{l}(X)=T_{l}(X) \otimes \mathbb{Q}_{l}$. Then

$$
H_{\text {èt }}^{2}\left(X, \mathbb{Q}_{l}\right)=V_{l}(X) \oplus\left(N S(X) \otimes \mathbb{Q}_{l}\right) .
$$

Let $\varphi(T)$ be the characteristic polynomial of $F^{*} \mid V_{l}(X)$. It is a polynomial over $\mathbb{Q}$ and equal to the characteristic polynomial of $F^{*} \mid T_{\text {cris }}(X)$ ([8], 3.7.3). Let $s_{1}, s_{2}, \ldots, s_{r}$ be the roots of $\varphi(T)$. After replacing $\mathbb{F}_{q}$ by a suitable finite extension, we may assume that if $s_{i} / s_{j}$ is a root of unity, then $s_{i}=s_{j}$. Let $\alpha$ be an automorphism of $X$. We may assume that $\alpha$ is defined over $\mathbb{F}_{q}$ after replacing the base field $\mathbb{F}_{q}$ by a finite extension. In this case, $F \circ \alpha=\alpha \circ F$. Since $F^{*}$ and $\alpha^{*}$ are semisimple on $V_{l}(X)$ [6], there exists a basis of $V_{l}(X)$ consisting of common eigenvectors for $F^{*}$ and $\alpha^{*}$. We assume that $t_{1}, \ldots, t_{r}$ are eigenvalues of $\chi_{l, X}(\alpha)$ and $s_{1} t_{1}, \ldots, s_{r} t_{r}$ are eigenvalues of $F^{*} \circ \alpha^{*} \mid V_{l}(X)$. Let $\psi(T) \in \mathbb{Q}[T]$ be the characteristic polynomial of $F^{*} \circ \alpha^{*} \mid T_{l}(V)$. Let us fix an embedding $\overline{\mathbb{Q}} \hookrightarrow \bar{K}$. There is a unique $q$-adic order $\operatorname{ord}_{q}(\cdot)$ on $\overline{\mathbb{Q}}$ associated to this embedding.

Because the height of $X$ is $h$, exactly $h$ roots of $\varphi(T)$ have order $1-1 / h$ for the $q$-adic order $\operatorname{ord}_{q}(\cdot)$. Assume that $\operatorname{ord}_{q}\left(s_{i}\right)=1-1 / h$ for $i=1, \ldots, h$. Then $s_{1}, \ldots, s_{h}$ are roots of characteristic polynomial of $F^{*} \mid H^{2}\left(X, W \mathcal{O}_{X}\right)$. We assume that $\rho_{X}(\alpha)=1$. By the proof of Theorem 3.9, $\alpha^{*} \mid H^{2}\left(X, W \mathcal{O}_{X}\right)=i d$. Because the characteristic polynomial of $(F \circ \alpha)^{*} \mid V_{l}(X)$ is equal to the characteristic polynomial of $(F \circ \alpha)^{*} \mid T_{\text {cris }}(X)$, if $\operatorname{ord}_{q}\left(s_{i}\right)<1$, then $t_{i}=1$. Now assume 
that $t_{i} \neq 1$ for some $i>h$. Because the Tate conjecture is valid for K3 surfaces [23; 19], $s_{i}$ is conjugate to $s_{j}$ over $\mathbb{Q}$ for some $j \leq h$. Suppose $\tau\left(s_{i}\right)=s_{j}$ for some $\tau \in \operatorname{Gal}(\overline{\mathbb{Q}} / \mathbb{Q})$. Then

$$
\tau\left(s_{i} t_{i}\right)=s_{j} \tau\left(t_{i}\right)=s_{k} t_{k}=s_{k}
$$

for some $k \leq h$. But it is impossible since $\tau\left(t_{i}\right) \neq 1$ is a root of unity. Therefore, $\chi_{l, X}(\alpha)=i d$ and $p_{l, X}: \operatorname{Im} \chi_{l, X} \rightarrow \operatorname{Im} \rho_{X}$ is injective.

COROLlARY 3.11. Let $k$ be an algebraic closure of a finite field of odd characteristic $p$, and $X$ be a K3 surface of finite height over $k$. If $N$ is the order of $\operatorname{Im} \rho_{X}$, then the rank of $T_{l}(X), 22-\rho(X)$ is divisible by $\phi(N)$.

Proof. We choose $\alpha \in \operatorname{Aut}(X)$ such that the order of $\rho_{X}(\alpha)$ is $N$. Assume that the order of $\chi_{l, X}(\alpha)$ is $p^{m} n$ for some nonnegative integers $m$ and $n$ where $p$ does not divide $n$. Since the order of $\rho_{X}\left(\alpha^{p^{m}}\right)$ is still $N$, replacing $\alpha$ by $\alpha^{p^{n}}$, we may assume that the order of $\chi_{l, X}(\alpha)$ is not divisible by $p$. Let $t_{i}$ be an eigenvalue of $\chi_{l, X}(\alpha)$. By the proof of Theorem $3.10, t_{i}$ is a primitive $N$ th root of unity, and $n$ is equal to $N$. It follows that the characteristic polynomial of $\chi_{l, X}(\alpha)$ is a power of the $N$ th cyclotomic polynomial over $\mathbb{Q}$ and the rank of $T_{l}(X)$ is a multiple of $\phi(N)$.

\section{Nonsymplectic Automorphism of Some High Order and Frobenius Invariant}

Proposition 4.1. Let $k$ be an algebraic closure of a finite field of odd characteristic $p$, and $X$ be a K3 surface over $k$. Let $\alpha$ be an automorphism of $X$. We assume that the order of $\rho_{X}(\alpha)$ is $N(>2)$ and that the rank of the Neron-Severi group of $X$ is at least $22-\phi(N)$. If $p^{m} \equiv-1$ modulo $N$ for some $m$, then $X$ is supersingular. If $p^{m} \not \equiv-1$ modulo $N$ for any $m$ and the order of $p$ in $(\mathbb{Z} / N \mathbb{Z})^{*}$ is $n$, then the height of $X$ is $n$.

Proof. Assume that $p^{m} \not \equiv-1$ modulo $N$ for any $m$. Then by Proposition 3.3, $X$ is of finite height.

We assume that $X$ is of finite height. Then, by the assumption and Corollary 3.11, the rank of $T_{l}(X)$ is $\phi(N)$, and the order of $\chi_{l, X}(\alpha)$ is equal to the order of $\rho(\alpha)$. Every eigenvalue of $\chi_{l, X}(\alpha)$ is a primitive $N$ th root of unity, and the characteristic polynomial of $\chi_{l, X}(\alpha)$ is the $N$ th cyclotomic polynomial over $\mathbb{Q}$. We denote the $N$ th cyclotomic polynomial over $\mathbb{Q}$ by $\Phi_{N}(T)$. Let $\zeta=\rho_{X}(\alpha)$, and let $\xi$ be the Teichmüller lift of $\xi$ in $W$. Let $V_{l}(X)=T_{l}(X) \otimes \mathbb{Q}_{l}$. Since every primitive $N$ th root of unity appears once as an eigenvalue of $\chi_{l, X}(\alpha), V_{l}(X)$ is a rank 1 free module over $\mathbb{Q}_{l}[T] / \Phi_{N}(T)$ by the action of $\alpha^{*}$. Note that

$$
\mathbb{Q}_{l}[T] / \Phi_{N}(T)=\left(\mathbb{Q}[T] / \Phi_{N}(T)\right) \otimes \mathbb{Q}_{l} \simeq \bigoplus_{k} \mathbb{Q}_{l}\left(\xi^{a_{k}}\right)
$$

for suitable primitive $N$ th roots of unity $\xi^{a_{k}}$. Suppose that $X$ and $\alpha$ are defined over $\mathbb{F}_{q}$ for $q=p^{r}$ and $F: X \rightarrow X$ is the $r$-iterative relative Frobenius morphism of $X / k$. Let $\varphi(T) \in \mathbb{Q}[T]$ be the characteristic polynomial of $F^{*} \mid V_{l}(X)$. Since 
$F^{*} \circ \alpha^{*}=\alpha^{*} \circ F^{*}, F^{*} \mid V_{l}(X)$ is a $\mathbb{Q}_{l}[T] / \Phi_{N}(T)$-module endomorphism, so it is the multiplication by a unit element of $\mathbb{Q}_{l}[T] / \Phi_{N}(T)$. Hence, all the roots of $\varphi(T)$ are contained in $\mathbb{Q}_{l}(\xi)$ for any $l \neq p$. It follows that, by the Chebotarev density theorem, all the roots of $\varphi(T)$ are contained in $\mathbb{Q}(\xi)$. Let $n$ be the order of $p$ in $(\mathbb{Z} / N \mathbb{Z})^{*}$. Then $\xi^{p^{-n}}=\xi$. Since a primitive $N$ th root of unity appears only once in the eigenvalues of $\alpha^{*} \mid T_{l}(X)$, by Theorem 3.9, the height of $X$ is at most $n$. We fix a $q$-adic order $\operatorname{ord}_{q}(\cdot)$ on $\overline{\mathbb{Q}}$. For a root of $\varphi(T), s \in \mathbb{Q}(\xi)$, by the Tate conjecture, there is $\tau \in \operatorname{Gal}(\overline{\mathbb{Q}} / \mathbb{Q})$ such that $\operatorname{ord}_{q}(\tau(s))<1$. Since $\operatorname{deg} \varphi(T)$ is $\phi(N)$ and the number of primes of $\mathbb{Q}(\xi)$ dividing $p$ is $\phi(N) / n$, the number of roots of $\varphi(T)$ whose $\operatorname{ord}_{q}(\cdot)$ orders are less than 1 is at least $\phi(N) /(\phi(N) / n)=n$. Therefore, the height of $X$ is at least $n$, so the height of $X$ is $n$. Now suppose $n=2 m$ and $p^{m} \equiv-1$ modulo $N$. Then the height of $X$ is $2 m$. But among $\xi^{ \pm 1}, \xi^{ \pm p^{-1}}, \ldots, \xi^{ \pm p^{-2 m+1}}, \xi$ appears twice as an eigenvalue of $\chi_{l, X}(\alpha)$. It contradicts to the assumption, and $X$ is supersingular.

REMARK 4.2. In the statement of the theorem, the assumption that the rank of the Neron-Severi group is at least $22-\phi(N)$ is satisfied if $\phi(N)>10$ by Corollary 3.11 .

COROLlary 4.3. Let $k$ be an algebraically closed field of odd characteristic $p$, and $X$ be a K3 surface over $k$. Let $\alpha$ be an automorphism of $X$. We assume that the order of $\rho_{X}(\alpha)$ is $N(>2)$ and that the rank of the Neron-Severi group of $X$ is at least $22-\phi(N)$.

(1) If $p^{m} \equiv-1$ modulo $N$ for some $m$, then $X$ is supersingular.

(2) If $p^{m} \not \equiv-1$ modulo $N$ for any $m$ and the order of $p$ in $(\mathbb{Z} / N \mathbb{Z})^{*}$ is $n$, then the height of $X$ is $n$.

Proof. There exists an integral model $\mathcal{X} / R$ of $X / k$, where $R$ is a Noetherian domain of finite type over $\mathbb{F}_{p}$ equipped with an embedding $R \hookrightarrow k$ such that a geometric generic fiber $k \otimes_{R} \mathcal{X}$ is isomorphic to $X / k$. After shrinking the base Spec $R$, we may assume that $N S(X)$ and $\alpha$ extend to $\mathcal{X} / R$. But the locus of degeneration of the Frobenius invariant is closed ([2], Sect. 8), so we may assume that every geometric fiber of $\mathcal{X} / R$ has the same Frobenius invariant as the generic fiber. We choose a closed fiber $X_{0}$ of $\mathcal{X} / R$, which is a K3 surface defined over a finite field. By the assumption, the rank of the Neron-Severi group of $X_{0} \otimes \overline{\mathbb{F}}_{p}$ is at least $22-\phi(N)$. Then the claim follows by Proposition 4.1.

COROLlaRY 4.4. Let $k$ be an algebraically closed field of odd characteristic $p$. Assume that $X$ is a K3 surface over $k$ and $\alpha$ is an automorphism of $X$ such that the order of $\rho_{X}(\alpha)$ is $N(>2)$. We assume that $\alpha$ is of finite order prime to $p$ and that a primitive $N$ th root of unity appears only once in the eigenvalues of $\alpha^{*} \mid H_{\text {et }}^{2}\left(X, \mathbb{Q}_{l}\right)$. If the order of $p$ in $(\mathbb{Z} / N \mathbb{Z})^{*}$ is $2 n$ and $p^{n} \equiv-1$ modulo $N$, then $X$ is supersingular of Artin-invariant $n$.

Proof. By the proof of Corollary 4.3, $X$ is supersingular. Since $n$ is the least number satisfying $p^{n} \equiv-1$ modulo $N$, the Artin-invariant of $X$ is at least $n$ by 
Proposition 3.3. On the other hand, by Theorem 3.9, the Artin-invariant of $X$ cannot be greater than $n$, so it is equal to $n$.

Because a supersingular K3 surface of Artin-invariant 1 is unique up to isomorphism, we obtain the following.

COROLlaRY 4.5. Let $k$ is an algebraically closed field of odd characteristic p. If $10<\phi(N)<22, N \neq 60$, and $p \equiv-1$ modulo $N$, then there exists a unique K3 surface over $k$ up to isomorphism that has a purely nonsymplectic automorphism of order $N$.

Proof. The existence can be checked in Section 3 of [13].

REMARK 4.6. Over $\mathbb{C}$, a K3 surface equipped with a purely nonsymplectic automorphism of some high order is unique $[18 ; 24 ; 1 ; 29]$. Also, there is a unique $\mathrm{K} 3$ surface with an automorphism of order 60 in characteristic $\neq 2$, and there is a unique K3 surface with an automorphism of order 66 in characteristic $\neq 2,3[14$; $15]$.

Assume that $X$ is a complex algebraic K3 surface such that the order of $\operatorname{Im} \chi_{X}$ is $N(>2)$ and the rank of the transcendental lattice of $X$ is $\phi(N)$. By [26], Corollary 3.9.4, $X$ corresponds to a CM point in a moduli Shimura variety and is defined over a number field. We assume that $X, N S(X)$, and $\operatorname{Aut}(X)$ are defined over a number field $F$, and we fix a smooth projective integral model $X_{R}$ of $X$ over a $\operatorname{ring} R$, where $\operatorname{Spec} R$ is an affine open set of the affine scheme of the ring of integers of $F, \operatorname{Spec} \mathfrak{o}_{F}$. For each place $v \in \operatorname{Spec} R$, let $p_{v}$ be the residue characteristic of $v$. We may assume that $p_{v} \nmid N d(N S(X))$ and $p_{v}$ is unramified in $F$ for any $v \in \operatorname{Spec} R$. We denote the reduction of $X_{R}$ over an algebraic closure of the residue field $k(v)$ by $X_{v}$.

Theorem 4.7. If $p_{v}^{m} \not \equiv-1$ modulo $N$ for all $m \in \mathbb{Z}$, then $X_{v}$ is of finite height, and the height of $X_{v}$ is the order of $p_{v}$ in $(\mathbb{Z} / N)^{*}$. If the order of $p_{v}$ in $(\mathbb{Z} / N)^{*}$ is $2 m$ and $p_{v}^{m} \equiv-1$ modulo $N$, then $X_{v}$ is supersingular of Artin-invariant $m$.

Proof. There is an embedding

$$
N S(X) \hookrightarrow N S\left(X_{v}\right),
$$

so the rank of $N S\left(X_{v}\right)$ is at least $22-\phi(N)$. By Corollary $4.3, p_{v}^{m} \not \equiv-1$ modulo $N$ for any $m \in Z$ if and only if $X_{v}$ is of finite height, and in this case, the height is equal to the order of $p_{v}$ in $(\mathbb{Z} / n \mathbb{Z})^{*}$.

Now assume that $X_{v}$ is supersingular and $2 m$ is the order of $p_{v}$ in $(\mathbb{Z} / N)^{*}$. We fix an automorphism $\alpha \in \operatorname{Aut}(X)$ such that $\xi=\rho_{X}(\alpha)$ is a primitive $N$ th root of unity. Note that we do not assume that $\alpha$ is of finite order. Let $T_{N S}(X)$ be the orthogonal complement of the embedding

$$
N S(X) \otimes W \hookrightarrow N S\left(X_{v}\right) \otimes W .
$$

Here $W$ is the ring of Witt vectors of the algebraic closure of $k(v)$. Because $N S\left(X_{v}\right) \otimes K$ is canonically isomorphic to $H_{d r}^{2}\left(X_{R} / R\right) \otimes K, \alpha^{*} \mid T_{N S}(X)$ is of finite 
Table 1

\begin{tabular}{ll}
\hline \hline Congruence class of $p_{v}$ modulo 36 & Frobenius invariant of $X_{v}$ \\
\hline 1 & ordinary \\
17 & height 2 \\
13,25 & height 3 \\
$5,7,19,29,31$ & height 6 \\
35 & supersingular of Artin-invariant 1 \\
11,23 & supersingular of Artin invariant 3 \\
\hline
\end{tabular}

order, and every $N$ th root of unity appears once as an eigenvalue of $\alpha^{*} \mid T_{N S}(X)$. Since $p$ does not divide $d(N S(X)), N S(X) \otimes W$ is unimodular. Because there is a unimodular sublattice of $N S\left(X_{v}\right) \otimes W$ of rank $22-\phi(N)$, the Artin-invariant of $X_{v}$ is at most $\phi(N) / 2$. If $\sigma$ is the Artin-invariant of $X_{v}$, then $N$ divides $p^{\sigma}+1$, so $p^{\sigma} \equiv-1$ modulo $N$, and $\sigma$ is an odd multiple of $n$. We have an inclusion

$$
N S\left(X_{v}\right)^{*} / N S\left(X_{v}\right) \simeq T_{N S}(X)^{*} / T_{N S}(X) \subseteq T_{N S}(X) / p T_{N S}(X),
$$

which is compatible with the actions of $\operatorname{Aut}(X)$. All the eigenvalues of $\alpha^{*} \mid\left(T_{N S}(X) / p T_{N S}(X)\right)$ are distinct. But if $\sigma$ is greater than $n$, then $\rho_{X_{v}}(\alpha)$ appears more than once in the eigenvalues of $\alpha^{*} \mid\left(N S\left(X_{v}\right)^{*} / N S\left(X_{v}\right)\right)$ by Theorem 3.9. This contradicts the assumption. Therefore, the Artin-invariant of $X_{v}$ is $n$.

EXAMPLE 4.8 (cf. [28; 30]). Let $X$ be a K3 surface defined over a number field $F$ such that the order of $\operatorname{Im} \rho_{X}$ is 36 . The rank of the transcendental lattice of $X$, $T(X)$ is $12=\phi(36)$. For example, an elliptic $\mathrm{K} 3$ surface $X_{36} / \mathbb{Q}$ defined by the equation

$$
y^{2}=x^{3}+t^{5}\left(t^{6}-1\right)
$$

has a purely nonsymplectic automorphism of order $36,(t, x, y) \mapsto\left(\xi^{30} t\right.$, $\xi^{2} x, \xi^{3} y$ ), where $\xi$ is a primitive 36 th root of unity. Although it is quite believable, we do not know whether $X_{36}$ is a unique complex K3 surface satisfying this condition. For almost all places $v$ of $F, X$ has a good reduction $X_{v}$. The Frobenius invariant of $X_{v}$ is in Table 1 .

Acknowledgment. The author thanks J. Keum and D. Lee for helpful comments.

\section{References}

[1] M. Artebani, A. Sarti, and S. Taki, K3 surfaces with non-symplectic automorphisms of prime order, Math. Z. 268 (2011), 507-533.

[2] M. Artin, Supersingular K3 surfaces, Ann. Sci. Éc. Norm. Supér. (4) 7 (1974), 543 567. 
[3] M. Artin and B. Mazur, Formal groups arising from algebraic varieties, Ann. Sci. Éc. Norm. Supér. (4) 10 (1977), 87-131.

[4] P. Brethelot and A. Ogus, F-isocrystals and de Rham cohomology I, Invent. Math. 72 (1983), 159-199.

[5] F. Charles, The Tate conjecture for K3 surfaces over finite fields, Invent. Math. 194 (2013), 119-145.

[6] P. Deligne, La conjecture de Weil pour les surfaces K3, Invent. Math. 15 (1972), 237-250.

[7] Y. Goto, A note on the height of the formal Brauer group of a K3 surface, Canad. Math. Bull. 47 (2004), no. 1, 22-29.

[8] L. Illusie, Report on crystalline cohomology, Algebraic Geometry, Proc. Sympos. Pure Math. 29 (1975), 459-478.

[9] Complexe de de Rham-Witt et cohomologie cristalline, Ann. Sci. Éc. Norm. Supér. (4) 12 (1979), 501-661.

[10] J. Jang, Neron-Severi group preserving lifting of $K 3$ surfaces and applications, Math. Res. Lett. 22 (2015), 789-802.

[11] _ Representations of the automorphism group of a supersingular K3 surface of Artin-invariant 1 over odd characteristic, J. Chungcheong Math. Soc. 27 (2014), no. 2, 287-295.

[12] N. Katz, Slope filtration of F-crystal, Astérisque 63 (1979), 113-163.

[13] J. Keum, Orders of automorphisms of K3 surfaces, arXiv:1203.5616.

[14] __ K3 surfaces with an order 60 automorphisms and a characterization of supersingular K3 surfaces with Artin invariant 1, Math. Res. Lett. 21 (2014), no. 3, 509-520.

[15] _ K3 surfaces with an automorphism of order 66, the maximum possible, J. A1gebra 426 (2015), 273-287.

[16] S. Kondo and I. Shimada, On certain duality of Neron-Severi lattices of supersingular K3 surfaces, arXiv:1212.0269.

[17] M. Lieblich and D. Maulik, A note on the cone conjecture for K3 surfaces in positive characteristic, arXiv:1102.3377.

[18] N. Machida and K. Oguiso, On K3 surfaces admitting finite non-symplectic group actions, J. Math. Sci. Univ. Tokyo 5 (1998), 273-297.

[19] K. Madapusi Pera, The Tate conjecture for K3 surfaces in odd characteristic, arXiv:1301.6326.

[20] D. Maulik, Supersingular K3 surfaces for large primes, Duke Math. J. 163 (2014), no. 13, 2357-2425.

[21] V. V. Nukulin, Finite groups of automorphisms of Kählerian K3 surfaces, Tr. Mosk. Mat. Obs. 38 (1979), 75-137.

[22] N. O. Nygaard, Higher de Rham-Witt complexes of supersingular K3 surfaces, Compos. Math. 42 (1981), 245-271.

[23] N. O. Nygaard and A. Ogus, Tate conjecture for K3 surfaces of finite height, Ann. of Math. (2) 122 (1985), 461-507.

[24] K. Oguiso and D. Zhang, On Vorontsov's theorem on K3 surfaces with non-symplectic group actions, Proc. Amer. Math. Soc. 128 (2000), 1571-1580.

[25] A. Ogus, A crystalline Torelli theorem for supersingular K3 surfaces, Progr. Math. 36 (1983), 361-394.

[26] J. Rizov, Kuga-Satake abelian varieties of K3 surfaces in mixed characteristic, J. Reine Angew. Math. 648 (2010), 13-67. 
[27] A. N. Rudakov and I. R. Shafarevich, Surfaces of type K3 over fields of finite characteristic, Current problems in mathematics, Vol. 18, pp. 115-207, Akad. Nauk SSSR, Vsesoyuz. Inst. Nauchn. i Tekhn. Informatsii, 1981.

[28] T. Shioda, Supersingular K3 surfaces with big Artin invariant, J. Reine Angew. Math. 381 (1987), 205-210.

[29] S. Taki, On Oguiso's K3 surface, J. Pure Appl. Algebra 218 (2014), 391-394.

[30] N. Yui, Formal Brauer groups arising from certain weighted K3 surfaces, J. Pure Appl. Algebra 142 (1999), 271-296.

Department of Mathematics

University of Ulsan

Daehakro 93

Namgu Ulsan 680-749

Korea

jmjang@ulsan.ac.kr 